\title{
Characteristics of cervical disease among symptomatic women with histopathological sample at Hawassa University referral hospital, Southern Ethiopia
}

Gemechu Ameya ${ }^{{ }^{*}}$ (D) and Fekade Yerakly ${ }^{2}$

\begin{abstract}
Background: Cervical cancer is one of the most common cancers of women in developing countries. It is also eminent cause of mortality and morbidity in women worldwide. Symptoms usually develop when the cancer has become invasive and invade nearby tissue. This has significant effect on treatment of cases in area where there is limited awareness. The aim of this study is to describe cervical disease among symptomatic women with a histhological sample at Hawassa University referral hospital, southern Ethiopia.

Methods: Five years retrospective histopathological characteristics of symptomatic cervical cases were studied from January, 2010 to January, 2015 at Hawassa University referral hospital pathology laboratory. Clinical diagnosis of patients, patient's age, biopsy sample size and type, and microscopic finding of the cases were collected for this study. The data were entered by Epi-Info statistical software version 7 and later exported to SPSS version 20 for analysis. Descriptive analyses such as frequency, mean, and standard deviation were computed.

Results: A total of 513 patients with cervical lesion were examined histopathologically in the study period. The age ranges of the patients were from 17 to 85 years with mean and standard deviation of $42 \pm 11$ years. Of these symptomatic examined cases, 253 (49.3\%) of them were cancerous lesion while non cancerous and precancerous cases account 166 (32.4\%) and 51 (9.9\%) respectively. Cervical squamous cell carcinoma was the predominant type cancer which accounts $211(83.4 \%)$ of all cancerous cervical cases. The proportion of cervical cancer was higher in age group older than 60 . The magnitude of cervical cancer and precancerous cases were steadily increasing throughout study periods whereas non cancerous cases were fluctuating.

Conclusions: Cervical cases were associated with wide range of disorders. Cervical cancer was the predominant cervical disease in symptomatic southern Ethiopian women. The high proportion of cervical cancer was observed in post-menopausal age when compared with other cervical cases. Effective uses of low-tech and inexpensive screening tools that already exist and giving awareness about the disease in combination with vaccine could reduce this high magnitude of cervical cancer in study area.
\end{abstract}

Keywords: Cervical cancer, Precancerous lesion, Histopathologic examination, Southern Ethiopia

\footnotetext{
* Correspondence: gemechuameya@gmail.com

${ }^{1}$ Department of Medical Laboratory Science, College of Medicine and Health

Sciences, Arba Minch University, P.O. Box: 21, Arba Minch, Ethiopia

Full list of author information is available at the end of the article
} 


\section{Background}

Cervical disease may range from the simple cervicitis to life treating cervical cancers. Cervical cancer is the most frequently occurring type of reproductive age cancers in women worldwide [1]. According to GLOBOCAN 2012 report, an estimated 14.1 million new cancer cases and 8.2 million cancer-related deaths occurred in 2012. Among these with 528,000 new cases every year, cervical cancer is the fourth most common cancer which is affecting women worldwide [2]. The burden of cervical cancer is quite low in the developed countries like North American with only 6.6 per 100,000 women new cervical cancer cases annually [2]. In contrast, it is most notable and cause of cancer death in the lower-resource countries of sub-Saharan Africa [1]. Almost 70\% of the global burden of cervical cancer falls in areas with lower levels of development [3]. In sub-Saharan Africa, about 34.8 new cases of cervical cancer case diagnosed per 100,000 women annually with 22.5 per 100,000 women die from the disease [1].

Similar to other east African countries, in Ethiopia cervical cancer is the most frequent cancer among women and it detected as early as 15 years of age [4]. According to resent WHO report, about 29.43 million women are at risk for cervical cancer. Annual cervical cancer cases were 7095 while 4732 deaths were observed due to cervical cancer. Crude incidence rate of cervical cancer was 16.3 per 100,000 populations [5]. This major difference with developed country may be due to lack of access to effective screening programs and to services that facilitate early detection and treatment as well as low level of awareness about the disease. This can have devastating effects with a very high social, human, and economic cost which affect women in their prime [6].

Cervical cancer arises from the cervix due to the abnormal growth of cells that have the ability to invade or spread to other parts of the body [6]. Histopathologic examination is the accurate method for diagnosis of different cervical lesions [7]. Carcinomas of the cervix can be categorized based on histological morphology into squamous cell carcinomas which is the predominant one, adenocarcinomas, adenosquamous carcinomas, and others [8]. Squamous cell cervical carcinoma tumors are also graded based on their degree of differentiation into well-differentiated, moderately-differentiated, and poorly-differentiated or undifferentiated [9].

Human papillomavirus (HPV) infections are the most common cause of cervical cancer through worldwide [4, 10]. Among more than 100 different types, infection with HPV 16 and 18 has been associated with more than $70 \%$ of cervical cancers [11]. Their type has not been associated either with survival or with morphological types of cancer. The virus can cause persistent infection and it will be risk for progression to precancerous lesion and cancer of the cervix $[4,10]$. Persistent HPV infection seems to be able to lead to preinvasive cancer (CIN III or cancer in situ) on its own, but cofactors are also required for development of invasive cancer [12].

Limited data are available on the HPV burden in the general population of Ethiopia. However, study conducted in Gondar, northwest Ethiopia indicates that about $92.6 \%$ of cervical cancer sample showed HPV positive. Among these, $76 \%$ of them are HPV type 16 [13]. According to WHO human papillomavirus and related diseases, prevalence of HPV was 97\%, 76.9\% and $26.5 \%$ among cervical cancer cases, high-grade and lowgrade precancerous lesions respectively. The predominant types of human papillomavirus in Ethiopia were HPV 16 and HPV 18 [5].

The study carried out in different part of Ethiopia showed that knowledge about cervical cancer is very low. In study that is conducted on northwest Ethiopia, only $31 \%$ of women were knowledgeable about cervical cancer [14]. From the findings of the studies that are done in Addis Ababa and southwest Ethiopia the awareness of people was very low. According to their thought, cervical cancer cause as a result of undertaking unacceptable behaviors or violating of social taboo [15]. Due to these reason patients seek medical care in advanced stage of cervical cancer which became difficult to treat.

According to recent WHO report, in Ethiopia national cervical cancer screening program is not established and screening coverage was only $0.8 \%$ [5]. In area where this research was done, the number of screening test conducted was very few, and histopathologic diagnosis was carried out for patient with symptom. We conduct this study on the formol saline fixed cervical biopsy sample taken at Hawassa University referral hospital and other its district hospitals. A routine hematoxylin and eosin staining technique was used to report histopathologic diagnosis. In this study area, so far there is no study conducted on histopathologic examination of cervical sample. Therefore, the aim of this study is to characterize cervical disease among symptomatic women with a histhological sample at Hawassa University referral hospital, southern Ethiopia.

\section{Methods}

Retrospective study was conducted on all cervical biopsy samples that were taken from symptomatic women of Hawassa district, southern Ethiopia from January, 2010 to January, 2015. Hawassa is the capital city of Southern Nations, Nationalities, and Peoples' region of Ethiopia. This region has a total population of 15,321,000 and nearly half of them were females according to the 2007 census report [16]. In this study area, cervical biopsy were taken and fixed with $10 \%$ formol saline fixative transported to Hawassa University referral hospital 
pathology laboratory for diagnosis. Upon receipt of all biopsy samples, identification number was given for each patient and the diagnosed results were recorded in both hardcopy and softcopy.

After the biopsy sample reached histopathology laboratory, a histopathology technicians give new laboratory number and ensure as fixative fluid was enough. Then senior pathologists examine the gross anatomy of the samples and take representative bits of the biopsy from the samples. The histopathology technicians of the laboratory processed these bits of tissues by the help of Leica TP 1020 tissue processor machine. During tissue processing, water was removed by using a number of increasing concentration of ethanol. This was followed by a hydrophobic clearing agent xylene to remove the alcohol and finally molten paraffin wax was used to infiltrate and replace the xylene. After that, the tissue was embedded by the help of Leica EG 1160 tissue embedding instrument so that the tissue in paraffin wax is firmly attached to tissue cassette. Rotary microtome was used to cut 3-5 $\mu \mathrm{m}$ section. Then the section was floated on the surface of water bath adjust at temperature just below the melting point of paraffin wax. This help to pick section on microscopic slide and to remove wrinkles appeared during sectioning. After the sectioned tissue was picked on microscopic slide, it was placed in a warm oven adjusted at $60{ }^{\circ} \mathrm{C}$ for about $30 \mathrm{~min}$ to so that it adheres to the slide. Then all cervical biopsy samples were stained with haematoxylin and eosin according to prepared standard operation procedure. Finally, the microscopic examination of stained slides was examined and reported by senior pathologist. Histopathologic classification of cervical cancer cases were also carried out according to the world health organization classification of cervical tumors [17].

For this study, we collected the information such as date and year of examination, clinical diagnosis of patient, patient's age, type of biopsy samples, biopsy sample size and site, and microscopic result of the samples. The gathered data was checked and entered by Epi-Info statistical software version 7 and later exported to SPSS version 20 for analysis. Descriptive analyses such as frequency, mean, and standard deviation were used.

\section{Results}

In the 5 years period (from January 2010 to January 2015), a total of 513 cervical biopsy samples were received from Hawassa University referral hospital pathology laboratory. The age range of patients was from 17 to 85 years with mean and standard deviation of $42 \pm 11$ SD. About 39\% of women with histopathologic sample were age group between 31 and 40 years old. Majority of samples 391 (76.2\%) taken in study period were punch biopsy.

Of 513 cervical biopsy examined in study period, only $43(8.4 \%)$ of them were reported as normal while the majority of them showed the same pathologic problems which range from simple cervicitis to cervical cancer. From all cervical cases, 253 (49.3\%) of symptomatic women were diagnosed for cervical cancer. Precancerous and non-cancerous cervical cases account 51 (9.9\%) and $166(32.4 \%)$ respectively (Table 1$)$.

About $80 \%$ of cervical cancer cases of the symptomatic women were observed in age above 60 years old while the least proportion was observed in age group less than 30 years old. In contrast, high proportion of non cancerous cases was observed in age group less than 30 , and low proportion was exhibited in older age. On the other hand, there was a little variation in proportion of precancerous cases between age groups, but its highest proportion was observed in age range between 41 and 50 years which accounts about $12 \%$ of cervical cases in the age group (Table 2).

In current study, squamous cell carcinoma (SCC) also accounts about $211(83.4 \%)$ of total cervical carcinoma is followed by adenocarcinoma which accounts 39 (15.4\%) and the others are adenosquamous carcinomas, and mucinous adenocarcinoma. Based on degree of differentiation, histological grading of SCC was also determined according to WHO grading, and moderately differentiated SCC was predominant one in study period. There was no great difference between proportions of grading of SCC; however; moderately differentiated SCC was high in all age groups (Table 3).

Chronic non-specific cervicitis which accounts $53.6 \%$ of non-cancerous cervical diseases was the second cause of cervical lesion. In the study period, Endocervical polyp was the second cause of non-cancerous cervical diseases. Furthermore, among precancerous cervical lesion, high grade cervical intraepithelial neoplasia was the predominant one.

Though out the study years, cervical cancer was the major cause of cervical disease in symptomatic women who were attending Hawassa University referral hospital. Its magnitude was also almost increasing in all study years except in 2013. In 2010, the magnitude of cervical cancer seems minimal but there was no great difference of proportion with other cervical disease in the study periods. Similarly, the trend of precancerous cervical lesion was increasing though out the study periods. On the other hand, a 5 year trend of non-cancerous cervical

Table 1 Numbers and percentages of cervical lesion in symptomatic women attending Hawassa University referral hospital $(n=513)$

\begin{tabular}{lll}
\hline Type of Cervical Lesion & Case number & In percent (\%) \\
\hline Precancerous cervical lesion & 51 & 9.9 \\
Cancerous cervical lesion & 253 & 49.3 \\
Non-cancerous cervical lesion & 166 & 32.4 \\
Normal cervical biopsy & 43 & 8.4 \\
Total & 513 & 100 \\
\hline
\end{tabular}


Table 2 Pattern of cervical lesion in symptomatic women attending Hawassa University referral hospital by age group ( $\mathrm{n}=513$ )

\begin{tabular}{llllll}
\hline Age of Patients & Precancerous cervical lesion & Cancerous cervical lesion & Non-cancerous cervical lesion & Normal cervical biopsy & Total (\%) \\
\hline$<30$ & $6(8.3 \%)$ & $24(33.3 \%)$ & $36(50 \%)$ & $6(8.3 \%)$ & $72(100 \%)$ \\
$31-40$ & $19(9.4 \%)$ & $84(41.8 \%)$ & $77(38.3 \%)$ & $31(10.4 \%)$ & $13(9.2 \%)$ \\
$41-50$ & $17(11.9 \%)$ & $74(52.1 \%)$ & $38(26.8 \%)$ & $2(2.7 \%)$ & $142(100 \%)$ \\
$51-60$ & $7(9.6 \%)$ & $51(69.9 \%)$ & $13(17.8 \%)$ & $1(4 \%)$ & $73(100 \%)$ \\
$>60$ & $2(8 \%)$ & $20(80 \%)$ & $2(8 \%)$ & $43(8.4 \%)$ & $25(100 \%)$ \\
Total & $51(9.9 \%)$ & $253(49.3 \%)$ & $166(32.4 \%)$ & $513(100 \%)$ \\
\hline
\end{tabular}

lesion was fluctuating in the study years. In 2012, the magnitude of non-cancerous cases was high whereas the lowest proportion with other cervical diseases was observed in 2014 (Fig. 1).

\section{Discussion}

Nowadays, more than twice as many people die from cancer than from infectious diseases. New cancer cases and deaths are also more common in low and middleincome countries. Cervical cancer is the second killer cancer of women next to breast cancer. In our study, it is also the predominant cervical diseases which account $49.32 \%$ in symptomatic women. Cervical cancers do not formed suddenly but the normal cervical cells gradually develop pre-cancerous changes that turn into cancer. Current study shows that about $10 \%$ of cases were precancerous change. This indicates that the magnitude of precancerous cases were by far less than cancerous cases. The possible reason for this discrepancy may be due to less awareness of cervical cancer screening in the area and women also visit the hospital after symptom is observed [14, 15].

In our study, the magnitude of cervical cancer in postmenopausal (age group above 50 years old) women was about $72 \%$ of cervical disease in symptomatic women. This finding was higher than the finding of the study conducted in Addis Ababa that was $51.6 \%$ of postmenopausal bleeding of patient [18]. The differences may be due to the restricted samples to those from women who have postmenopausal bleeding. Furthermore, the observed difference may be due to the difference in socio- demographic status of the patient and difference in the level of awareness between study populations.

In this study, SCC was the predominant type of cancer which accounts about $83 \%$ of cervical cancer. Unlike our finding, study conducted in Malaysia showed that SCC was the second cervical lesion with $29 \%$ of cervical lesion next to precancerous cervical lesion which account $42 \%$ of cervical lesion [19]. The observed difference may be because of little awareness and screening in Ethiopia and most lesions are already cancerous when diagnosed whereas in other places like Malaysia, where screening is undertaken, this was not the case [19].

Adenocarcinoma encountered about $15 \%$ of the cancerous cases of symptomatic women in current study. Some authors showed that adenocarcinoma was associated with a worse prognosis when compared to SCC [20]. Magnitude of precancerous cervical lesion and poorly-differentiated SCC were less than moderately differentiated and well differentiated SCC. According to study conducted at the University of Michigan, patients with a well-differentiated tumor had more survival rate (Survival rate $85 \%$ ) than those with a poorly differentiated tumor (Survival rate 57\%) [21]. Other major determinant for the prognosis of cervical cancer is the stage at which the patient present. The study also shows that most patients in developing countries including Ethiopia present late with advanced stage disease [22]. This markedly diminished chance of success of treatment and study also indicates that there is high death rate in later stage of cervical cancer $[5,23]$. In this study, histological classification of SCC was made according to WHO

Table 3 Distribution of cervical squamous cell carcinoma by histological grading and age group in symptomatic women attending Hawassa University referral hospital $(n=211)$

\begin{tabular}{|c|c|c|c|c|}
\hline \multirow{2}{*}{$\begin{array}{l}\text { Age of } \\
\text { patient }\end{array}$} & \multicolumn{3}{|c|}{ Histological grading of SCC } & \multirow[b]{2}{*}{ Total (\%) } \\
\hline & Poorly-differentiated & Moderately-differentiated & Well-differentiated & \\
\hline$<30$ & $6(35.3 \%)$ & $9(52.9 \%)$ & $2(11.8 \%)$ & $17(100 \%)$ \\
\hline $31-40$ & $11(15.3 \%)$ & $33(45.8 \%)$ & $28(38.9 \%)$ & 72 (100\%) \\
\hline $41-50$ & $12(19.4 \%)$ & $28(45.2 \%)$ & $22(35.5 \%)$ & $62(100 \%)$ \\
\hline $51-60$ & $9(21.9 \%)$ & $19(46.3 \%)$ & $13(31.7 \%)$ & $41(100 \%)$ \\
\hline$>60$ & $7(36.8 \%)$ & $9(47.4 \%)$ & $3(15.8 \%)$ & $19(100 \%)$ \\
\hline Total & $45(21.3 \%)$ & 98 (46.4\%) & 68 (32.2\%) & 211 (100\%) \\
\hline
\end{tabular}




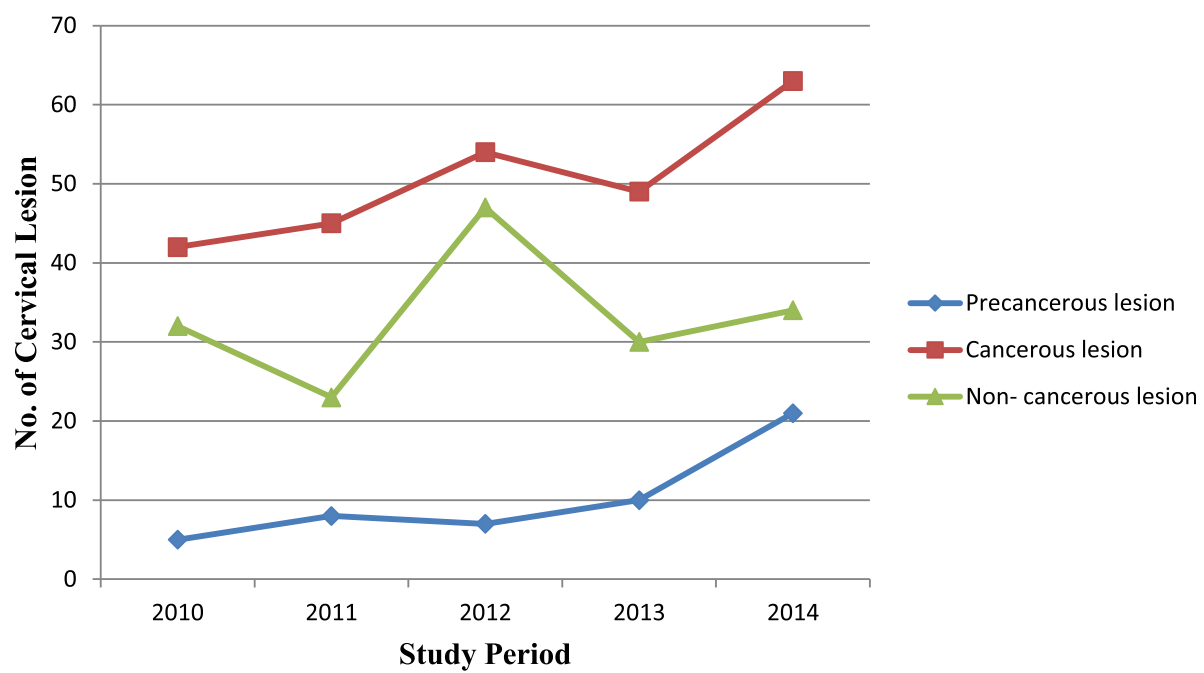

Fig. 1 The 5 years trend of cervical lesion in symptomatic women attending Hawassa University referral hospital

histological grading. The histological differentiation stage is strongly associated with tumor behavior. Usually poorly-differentiated is more aggressive than the well differentiated counterpart $[24,25]$. The histological classification is intended to facilitate the comparison of results and outcomes. It is also useful to pathologists, gynecologist, radiotherapists, oncologists, and epidemiologists in classification and characterization of cancer. Again, it is important for a reliable prognosis to be established and it provides the basis for clinical management of patients. As observed in our study, majority of malignant lesion of the cervix were squamous cell carcinomas, but other tumor types must be distinguished pathologically because different phenotypes have different biological behavior $[8,9]$.

In this study, the minimum age of the patient in which cervical cancer was reported was 17 years old. In the other study which is conducted in Ethiopia, cervical cancer was detected in 15 years old [4]. As a whole, the proportion of cervical cancer increases with age of the patient whereas non-cancerous cervical lesion was inversely proportional with age. In developing countries for example, Ethiopia where there is no well-organized screening program, this pattern is expected and the only way to reverse that is by investing in screening and awareness. Study also shows that one-third of cancer deaths can be prevented through screening tests, vaccinations, and lifestyle changes [26].

In this study, the magnitude of precancerous cervical cancerous lesion was $10 \%$ which is comparable with study done in Nigeria (6\%) [27]. High prevalence of HPV and human immunodeficiency virus (HIV) in subSaharan countries can be contributor for the high rate of precancerous and cancerous cervical cases. In the study which is done in southern Ethiopia, HIV positive women, high prevalence $(22.1 \%)$ of precancerous cervical lesion was reported. The observed difference with our study is due to difference in study population. In their study, the patients are immunocompromised and they are susceptible for different infection disease including HPV. The other possible reason is most of precancerous lesion is asymptomatic and patient might not seek screening service unless they experience some sort of sign and symptom which is common in late stage of cancer in our case $[14,15]$.

In this study, the second predominant cervical disease was non-cancerous lesion; among them $53.6 \%$ of them was chronic nonspecific cervicitis. Similar study that is conducted in Nigeria, chronic nonspecific cervicitis was the most commonly encountered lesion constituting $72.2 \%$ of cervical inflammatory lesions [28]. Similarly, result that is observed in Puducherry, India was high which accounts $89.23 \%$ of cervicitis [29]. Cervicitis can be caused by different infectious pathogens which are transmitted by sexual intercourse. In this study, histopathological differential diagnosis of cervicitis was not performed. However, the study showed that the majority of cervicitis was caused by C. trachomatis, N. gonorrhoeae and herpes simplex virus [30]. In current study, the magnitude of endocervical polyp was $39.8 \%$ of noncancerous lesion, and it is about $13 \%$ of total cervical lesion which was higher than study conducted in Danish (7.8\%) [31].

Magnitude of cervical cancer was almost increasing in study periods. This may be due to increment in the level of awareness of women. Nowadays, as compared to previous time in Ethiopia, ministry of health also gives a great attention for this killer disease; as a result, women are initiated for screening. This may also the reason why the trend of precancerous cervical lesion was increasing 
throughout the study period. Non-cancerous cervical lesion was fluctuating in the study years and high magnitude was observed in 2012. The possible reason for this increment may be lack of well organized screening and control scheme in the study area, and women mainly screened in case experience symptom. In general, cervical cancer is remaining major health problem of southern Ethiopian women.

As limitation, our study is retrospective and based on histopatholagical sample obtained in pathology laboratory. Screening test of asymptomatic cases was very limited in study area and for this reason we consider only symptomatic cases. There was no data of histopathologic differentiation for adenocarcinoma. The way women get better awareness on the screening of cervical cancer should be searched so that women in study area can know their status before a lesion advanced.

\section{Conclusion}

Cervical diseases can be associated with wide range of disorders. It was the predominant cervical disease in symptomatic women who were attending Hawassa University referral hospital. In our study, in contrast to noncancerous cervical disease, the proportion of cervical cancer was high in older age. The magnitude of cervical precancerous changed and cervical cancer was increasing throughout study periods whereas non-cancerous cervical disease was fluctuating. Effective using of low-tech and inexpensive screening tools that already exist and giving awareness about the disease with vaccination could reduce this high magnitude of cervical cancer in study area.

\section{Abbreviations}

CIN: Cervical intraepithelial neoplasia; HIV: Human immunodeficiency virus; HPV: Human papillomavirus; SCC: Squamous cell carcinoma

\section{Acknowledgements}

We are grateful to Hawassa University, College of medicine and health sciences department of Pathology for permission of data to carry out this study and the staff of pathology laboratory for their assistance during data collection.

\section{Funding}

Not applicable.

\section{Availability of data and materials}

The datasets supporting the conclusions of this article are included within the article.

\section{Authors' contributions}

GA: participated in the design of the study, conducted data collection, analyzed the data and drafted the paper. FY: participated in the design of the study, analysis of the data and revised subsequent drafts of the paper. Both authors have read and approved the final manuscript.

\section{Ethics approval and consent to participate}

Institutional ethical clearance was obtained from research and ethics review board of Hawassa University, College of medicine and health sciences. A formal letter of cooperation was also written to obtain permission from the head of pathology department before using the data.
Consent for publication

Not applicable.

\section{Competing interests}

The authors declare that they have no competing interests.

\section{Publisher's Note}

Springer Nature remains neutral with regard to jurisdictional claims in published maps and institutional affiliations.

\section{Author details}

${ }^{1}$ Department of Medical Laboratory Science, College of Medicine and Health Sciences, Arba Minch University, P.O. Box: 21, Arba Minch, Ethiopia.

${ }^{2}$ Department of Pathology, School of medicine, College of medicine and health sciences, Hawassa University, Hawassa, Ethiopia.

Received: 22 August 2015 Accepted: 20 September 2017

Published online: 29 September 2017

References

1. Ferlay J, Soerjomataram I, Ervik M, Dikshit R, Eser S, Mathers C, Rebelo M, Parkin DM, Forman D, Bray F. GLOBOCAN 2012 v1.0, Cancer Incidence and Mortality Worldwide: IARC CancerBase No. 11 [Internet]. Lyon: International Agency for Research on Cancer; 2013. Available from http://globocan.iarc.fr. Accessed 10 July 2015

2. WHO. International Agency for research on cancer. Latest world cancer statistics. 2013. No. 223 Available on: http://www.iarc.fr/en/media-centre/pr/ 2013/pdfs/pr223_E.pdf Accessed 10 Aug 2015.

3. Bray F, Ren JS, Masuyer E, Ferlay J. Global estimates of cancer prevalence for 27 sites in the adult population in 2008. Int J Cancer. 2013;132(5):1133-45.

4. Parkin DM, Bray F, Ferlay J. Global cancer statistics. CA Cancer J Clin. 2005;55:74-108.

5. Bruni L, Barrionuevo-Rosas L, Albero G, Aldea M, Serrano B, Valencia S, et al. ICO Information Centre on HPV and Cancer (HPV Information Centre). Human Papillomavirus and related diseases in Ethiopia. 2016. Available on: http://www.hpvcentre.net/statistics/reports/XWX.pdf.

6. National Cancer Institute. Defining cancer. 2015. Available on: http://www. cancer.gov/cancertopics/cancerlibrary/what-is-cancer. Accessed 16 Aug 2015.

7. Kumar V, Abbas AK, Fausto N, Mitchell RN. Robbins Basic Pathology. 8th ed: Saunders Elsevier; 2007. p. 718-21.

8. Vizcaino AP, Moreno V, Bosch FX, Munoz N, Barros-Dios XM, Parkin DM. International trends in the incidence of cervical cancer: I. Adenocarcinoma and adenosquamous cell carcinomas. Int J Cancer. 1998;75(4):536-45.

9. Tiltman AJ. The pathology of cervical tumours. Best Pract Res Clin Obstet Gynaecol. 2005;19(4):485-500.

10. Trottier $\mathrm{H}$, Franco EL. Human papillomavirus and cervical cancer: burden of illness and basis for prevention. Am J Manag Care. 2006;12(17):462-72.

11. World Health Organization. Human papillomavirus (HPV) and cervical cancer. WHO Fact sheet. 2016. Available on: http://www.who.int/ mediacentre/factsheets/fs380/en/. Accessed on 08 Aug 2017.

12. Ferenczy A, Franco E. Persistent human papillomavirus infection and cervical neoplasia. Lancet Oncol. 2002;3(1):11-6.

13. Fanta BE. The distribution of Human PapillomaVirus infection in women with cervical histological abnormalities from an area with high incidence of cervical cancer. Ethiop Med J. 2005:43:151-8.

14. Getahun F, Mazengia F, Abuhay M, Birhanu Z. Comprehensive knowledge about cervical cancer is low among women in Northwest Ethiopia. BMC Cancer. 2013;13:2

15. Birhanu Z, Abdissa A, Belachew T, Deribew A, Segni H, Tsu, et al. Health seeking behavior for cervical cancer in Ethiopia: a qualitative study. Int J Equity Health. 2012;11:83.

16. Federal Democratic Republic of Ethiopia. Population census commission and UNFPA Summary and Statistical report of the 2007. Population and Housing Census. Addis Ababa, Ethiopia, 2008. Available on: https://www. scribd.com/doc/28289334/Summary-and-Statistical-Report-of-the-2007.

17. WHO histological classification of tumours of the uterine cervix. http:// screening.iarc.fr/atlasclassifwho.php. Accessed on 20 June 2015.

18. Ergete W, Tesfaye A. Histopathological findings of Post-Menopausal bleeding in Ethiopian women. Ethiop J Health Dev. 2001;15(1):39-44.

19. Karim A, Al-Naggar RA, San P, Mashani M. Histopathological findings for cervical lesions in Malaysian Women. Asian Pac J Cancer Prev. 2009;10:1159-62. 
20. Yamauchi M, Fukuda T, Wada T, Kawanishi M, Imai K, Hashiguchi Y. Comparison of outcomes between squamous cell carcinoma and adenocarcinoma in patients with surgically treated stage I-II cervical cancer. Mol Clin Oncol. 2014;2:518-24. doi: 10.3892/mco.2014.295.

21. Hopkins MP, Morley GW. Squamous cell cancer of the cervix: prognostic factors related to survival. Int J Gynecol Cancer. 1991;1(4):173-7.

22. Hamad HM. Cancer initiatives in Sudan. Ann Oncol. 2006;17(Suppl 8):viii32-6.

23. Kamangar F, Dores GM, Anderson WF. Patterns of cancer incidence, mortality, and prevalence across five continents: defining priorities to reduce cancer disparities in different geographic regions of the world. J Clin Oncol. 2006:24(14):2137-50.

24. Jogi A, Vaapil M, Johansson M, Pahlman S. Cancer cell differentiation heterogeneity and aggressive behavior in solid tumors. Ups J Med Sci. 2012; 117:217-24.

25. Sasieni P, Castanon A. NHSCSP audit of invasive cervical cancer: national report 2009-2013. 2014. Available on: http://www.wolfson.qmul.ac.uk/centrereports/centre-for-cancer-prevention. Accessed 28 Feb 2017.

26. Centers for disease control and prevention CDC 24/7 saving life. The global burden of cancer. Available on: http://www.cdc.gov/cancer/international/ burden.htm. Accessed 26 June 2015.

27. Ononogbu U, Almujtaba M, Modibbo F, Lawal I, Offiong R. Cervical cancer risk factors among HIV-infected Nigerian women. BMC Public Health. 2013;13:582.

28. Nwachokor FN, Forae GC. Morphological spectrum of non-neoplastic lesions of the uterine cenvix in Warri, South-South, Nigeria. Niger J Clin Pract. 2013;16:429-32.

29. Jayakumar NKB. Cervicitis: how often is it non specific. J Clin Diagn Res. 2015:9(3):EC11-2.

30. Centers for Disease Control and Prevention. Sexually transmitted diseases treatment guidelines, 2010: diseases characterized by urethritis and cervicitis. http://www.cdc.gov/std/treatment/2010/urethritis-and-cervicitis. htm. Accessed: 17 May 2015.

31. Dreisler E, Stampe Sorensen S, Ibsen PH, Lose G. Prevalence of endometrial polyps and abnormal uterine bleeding in a Danish population aged 20-74 years. Ultrasound Obstet Gynecol. 2009;33:102-8.

\section{Submit your next manuscript to BioMed Central and we will help you at every step:}

- We accept pre-submission inquiries

- Our selector tool helps you to find the most relevant journal

- We provide round the clock customer support

- Convenient online submission

- Thorough peer review

- Inclusion in PubMed and all major indexing services

- Maximum visibility for your research

Submit your manuscript at www.biomedcentral.com/submit

) Biomed Central 